Research Article

\title{
Convolution Properties for Some Subclasses of Meromorphic Functions of Complex Order
}

\author{
M. K. Aouf, ${ }^{1}$ A. O. Mostafa, ${ }^{1}$ and H. M. Zayed ${ }^{2}$ \\ ${ }^{1}$ Department of Mathematics, Faculty of Science, Mansoura University, Mansoura 35516, Egypt \\ ${ }^{2}$ Department of Mathematics, Faculty of Science, Menofia University, Shebin El Kom 32511, Egypt \\ Correspondence should be addressed to H. M. Zayed; hanaa_zayed42@yahoo.com
}

Received 3 June 2015; Accepted 5 July 2015

Academic Editor: Allan Peterson

Copyright (c) 2015 M. K. Aouf et al. This is an open access article distributed under the Creative Commons Attribution License, which permits unrestricted use, distribution, and reproduction in any medium, provided the original work is properly cited.

Making use of the operator $\mathscr{L}_{v}$ for functions of the form $f(z)=1 / z+\sum_{k=1}^{\infty} a_{k} z^{k-1}$, which are analytic in the punctured unit disc $\mathbb{U}^{*}=\{z: z \in \mathbb{C}$ and $0<|z|<1\}=\mathbb{U} \backslash\{0\}$, we introduce two subclasses of meromorphic functions and investigate convolution properties, coefficient estimates, and containment properties for these subclasses.

\section{Introduction}

Let $\Sigma$ denote the class of meromorphic functions of the form

$$
f(z)=\frac{1}{z}+\sum_{k=1}^{\infty} a_{k} z^{k-1},
$$

which are analytic in the punctured unit disc $\mathbb{U}^{*}=\{z: z \in \mathbb{C}$ and $0<|z|<1\}=\mathbb{U} \backslash\{0\}$. Let $g(z) \in \Sigma$ be given by

$$
g(z)=\frac{1}{z}+\sum_{k=1}^{\infty} b_{k} z^{k-1}
$$

then, the Hadamard product (or convolution) of $f(z)$ and $g(z)$ is given by

$$
(f * g)(z)=\frac{1}{z}+\sum_{k=1}^{\infty} a_{k} b_{k} z^{k}=(g * f)(z) .
$$

We recall some definitions which will be used in our paper.

Definition 1. For two functions $f(z)$ and $g(z)$, analytic in $\mathbb{U}$, we say that the function $f(z)$ is subordinate to $g(z)$ in $\mathbb{U}$ and written $f(z) \prec g(z)$, if there exists a Schwarz function $w(z)$, analytic in $\mathbb{U}$ with $w(0)=0$ and $|w(z)|<1$ such that $f(z)=g(w(z))(z \in \mathbb{U})$. Furthermore, if the function $g(z)$ is univalent in $\mathbb{U}$, then we have the following equivalence (see [1]):

$$
\begin{aligned}
f(z) & \prec g(z) \\
& \Longleftrightarrow f(0)=g(0), \\
f(\mathbb{U}) & \subset g(\mathbb{U}) .
\end{aligned}
$$

Now, consider Bessel's function of the first kind of order $v$ where $v$ is an unrestricted (real or complex) number, defined by (see Watson [2, page 40]) (see also Baricz [3, page 7])

$$
J_{v}(z)=\sum_{k=0}^{\infty} \frac{(-1)^{k}}{\Gamma(k+1) \Gamma(k+v+1)}\left(\frac{z}{2}\right)^{2 k+v},
$$

which is a particular solution of the second order linear homogenous Bessel differential equation (see, e.g., Watson [2, page 38]) (see also Baricz [3, page 7])

$$
z^{2} w^{\prime \prime}(z)+z w^{\prime}(z)+\left(z^{2}-v^{2}\right) w(z)=0 .
$$

Also, let us define

$$
\begin{aligned}
\mathscr{L}_{v}(z) & =\frac{2^{v} \Gamma(v+1)}{z^{v / 2+1}} J_{v}\left(z^{1 / 2}\right) \\
& =\frac{1}{z}+\sum_{k=1}^{\infty} \frac{(-1)^{k} \Gamma(v+1)}{4^{k} \Gamma(k+1) \Gamma(k+v+1)} z^{k-1}
\end{aligned}
$$


The operator $\mathscr{L}_{v}$ is a modification of the operator introduced by Szász and Kupán [4] for analytic functions.

By using the Hadamard product (or convolution), we define the operator $\mathscr{L}_{v}$ as follows:

$$
\begin{aligned}
\left(\mathscr{L}_{v} f\right)(z) & =\mathscr{L}_{v}(z) * f(z) \\
& =\frac{1}{z}+\sum_{k=1}^{\infty} \frac{(-1)^{k} \Gamma(v+1)}{4^{k} \Gamma(k+1) \Gamma(k+v+1)} a_{k} z^{k-1} .
\end{aligned}
$$

It is easy to verify from (8) that

$$
\begin{aligned}
z\left(\left(\mathscr{L}_{v+1} f\right)(z)\right)^{\prime}= & (v+1)\left(\mathscr{L}_{v} f\right)(z) \\
& -(v+2)\left(\mathscr{L}_{v+1} f\right)(z) .
\end{aligned}
$$

Definition 2. For $0 \leq \lambda<1,-1 \leq B<A \leq 1$, and $b \in$ $\mathbb{C}^{*}=\mathbb{C} \backslash\{0\}$, let $\Sigma \mathcal{S}_{\lambda}^{*}[b ; A, B]$ be the subclass of $\Sigma$ consisting of function $f(z)$ of the form (1) and satisfying the analytic criterion

$$
1+\frac{1}{b}\left[\frac{-z f^{\prime}(z)}{(1-\lambda) f(z)-\lambda z f^{\prime}(z)}-1\right] \prec \frac{1+A z}{1+B z} .
$$

Also, let $\sum \mathscr{K}_{\lambda}[b ; A, B]$ be the subclass of $\Sigma$ consisting of function $f(z)$ of the form (1) and satisfying the analytic criterion

$$
\begin{aligned}
1+ & \frac{1}{b}\left[\frac{-z\left(z f^{\prime}(z)\right)^{\prime}}{(1-\lambda) z f^{\prime}(z)-\lambda z\left(z f^{\prime}(z)\right)^{\prime}}-1\right] \\
& \prec \frac{1+A z}{1+B z} .
\end{aligned}
$$

It is easy to verify from (10) and (11) that

$$
\begin{aligned}
f(z) & \in \sum \mathscr{K}_{\lambda}[b ; A, B] \\
& \Longleftrightarrow-z f^{\prime}(z) \in \Sigma \mathcal{S}_{\lambda}^{*}[b ; A, B] .
\end{aligned}
$$

We note that

(i) $\sum \mathcal{S}_{0}^{*}[b ; A, B]=\sum \mathcal{S}^{*}[b ; A, B]$ and $\sum \mathscr{K}_{0}[b ; A, B]=$ $\sum \mathscr{K}[b ; A, B]$ (see Bulboacă et al. [5]);

(ii) $\sum \mathcal{S}_{0}^{*}[b ; 1,-1]=\Sigma \mathcal{S}(b)$ and $\Sigma \mathscr{K}_{0}[b ; 1,-1]=\sum \mathscr{K}(b)$ (see Aouf [6]);

(iii) $\Sigma \mathcal{S}_{0}^{*}\left[(1-\alpha) e^{-i \mu} \cos \mu ; 1,-1\right]=\Sigma \mathcal{S}^{\mu}(\alpha)$ and $\sum \mathscr{K}_{0}[(1-$ $\left.\alpha) e^{-i \mu} \cos \mu ; 1,-1\right]=\sum \mathscr{K}^{\mu}(\alpha)(\mu \in \mathbb{R},|\mu| \leq \pi / 2,0 \leq$ $\alpha<1$ ) (see Ravichandran et al. [7, with $p=1]$ ).

Definition 3. For $0 \leq \lambda<1,-1 \leq B<A \leq 1, b \in \mathbb{C}^{*}$ and $v$ is an unrestricted (real or complex) number, let

$$
\begin{aligned}
& \sum \mathcal{S}_{\lambda, v}^{*}[b ; A, B] \\
& \quad=\left\{f(z) \in \Sigma:\left(\mathscr{L}_{v} f\right)(z) \in \sum \mathcal{S}_{\lambda}^{*}[b ; A, B]\right\}, \\
& \sum \mathscr{K}_{\lambda, v}[b ; A, B] \\
& \quad=\left\{f(z) \in \sum:\left(\mathscr{L}_{v} f\right)(z) \in \sum \mathscr{K}_{\lambda}[b ; A, B]\right\} .
\end{aligned}
$$

It is easy to show that

$$
\begin{aligned}
f(z) & \in \sum \mathscr{K}_{\lambda, v}[b ; A, B] \\
& \Longleftrightarrow-z f^{\prime}(z) \in \sum \mathcal{S}_{\lambda, v}^{*}[b ; A, B] .
\end{aligned}
$$

The object of the present paper is to investigate some convolution properties, coefficient estimates, and containment properties for the subclasses $\sum \mathcal{S}_{\lambda, v}^{*}[b ; A, B]$ and $\sum \mathscr{K}_{\lambda, v}[b ; A, B]$.

\section{Main Results}

Unless otherwise mentioned, we assume throughout this paper that $0 \leq \lambda<1,-1 \leq B<A \leq 1, b \in \mathbb{C}^{*}$ and $v$ is an unrestricted (real or complex) number.

Theorem 4. If $f(z) \in \sum$, then $f(z) \in \sum \mathcal{S}_{\lambda}^{*}[b ; A, B]$ if and only if

$$
z\left[f(z) * \frac{1-[(\lambda-1) M+(\lambda+1)] z}{z(1-z)^{2}}\right] \neq 0
$$

$$
\text { for } z \in \mathbb{U} \text {, }
$$

where $M=M_{\theta}=\left(e^{-i \theta}+B\right) /(A-B) b, \theta \in[0,2 \pi)$, and also $M=0$.

Proof. It is easy to verify that

$$
\begin{gathered}
f(z) * \frac{1}{z(1-z)}=f(z), \\
f(z) *\left[\frac{1}{z(1-z)^{2}}-\frac{2}{(1-z)^{2}}\right]=-z f^{\prime}(z) \\
\forall z \in \mathbb{U}^{*} ; f \in \Sigma .
\end{gathered}
$$

(i) In view of (10), $f(z) \in \sum \mathcal{S}_{\lambda}^{*}[b ; A, B]$ if and only if (10) holds. Since the function $(1+[B+(A-B) b] z) /(1+B z)$ is analytic in $\mathbb{U}$, it follows that $(1-\lambda) f(z)-\lambda z f^{\prime}(z) \neq 0$ for $z \in \mathbb{U}^{*}$ or $z\left[(1-\lambda) f(z)-\lambda z f^{\prime}(z)\right] \neq 0$ for $z \in \mathbb{U}$; this is equivalent to (15) holdding for $M=0$. To prove (15) for all $M \neq 0$, we write (10) by using the principle of subordination as

$$
\frac{-z f^{\prime}(z)}{(1-\lambda) f(z)-\lambda z f^{\prime}(z)}=\frac{1+[B+(A-B) b] w(z)}{1+B w(z)},
$$

where $w(z)$ is Schwarz function, analytic in $\mathbb{U}$ with $w(0)=0$ and $|w(z)|<1$; hence,

$$
\begin{aligned}
& z\left[-z f^{\prime}(z)\left(1+B e^{i \theta}\right)-\left[(1-\lambda) f(z)-\lambda z f^{\prime}(z)\right]\right. \\
& \left.\cdot\left[1+[B+(A-B) b] e^{i \theta}\right]\right] \neq 0 \\
& \quad \text { for } z \in \mathbb{U}, \theta \in[0,2 \pi) .
\end{aligned}
$$


Using (16), (18) may be written as

$$
\begin{aligned}
& z[f(z) \\
& \left.\quad * \frac{1-\left[(\lambda-1)\left(\left(e^{-i \theta}+B\right) /(A-B) b\right)+(\lambda+1)\right] z}{z(1-z)^{2}}\right] \\
& \quad \neq 0 \text { for } z \in \mathbb{U} .
\end{aligned}
$$

Thus, the first part of Theorem 4 was proved.

(ii) Reversely, because assumption (15) holds for $M=0$, it follows that $z\left[(1-\lambda) f(z)-\lambda z f^{\prime}(z)\right] \neq 0$ for $z \in \mathbb{U}$. This implies that $\varphi(z)=-z f^{\prime}(z) /\left((1-\lambda) f(z)-\lambda z f^{\prime}(z)\right)$ is analytic in $\mathbb{U}$ (i.e., it is regular in $z=0$, with $\varphi(0)=1$ ).

Since it was shown in the first part of the proof that assumption (18) is equivalent to (15), we obtain that

$$
\frac{-z f^{\prime}(z)}{(1-\lambda) f(z)-\lambda z f^{\prime}(z)} \neq \frac{1+[B+(A-B) b] e^{i \theta}}{1+B e^{i \theta}}
$$

for $z \in \mathbb{U}, \theta \in[0,2 \pi)$.

Assume that

$$
\psi(z)=\frac{1+[B+(A-B) b] e^{i \theta}}{1+B e^{i \theta}} .
$$

Relation (20) means that $\varphi(\mathbb{U}) \cap \psi(\partial \mathbb{U})=\emptyset$. Thus, the simply connected domain is included in a connected component of $\mathbb{C} \backslash \psi(\partial \mathbb{U})$. From this, using the fact that $\varphi(0)=\psi(0)$ and the univalence of the function $\psi$, it follows that $\varphi(z) \prec \psi(z)$; this implies that $f(z) \in \sum \mathcal{S}_{\lambda, v}^{*}[b ; A, B]$. Thus, the proof of Theorem 4 is completed.

Remark 5. (i) Putting $\lambda=0$ in Theorem 4, we obtain the result obtained by Bulboacă et al. [5, Theorem 1].

(ii) Putting $\lambda=0, b=1$, and $e^{i \theta}=x$ in Theorem 4, we obtain the result obtained by Ponnusamy [8, Theorem 2.1].

(iii) Putting $\lambda=0, b=(1-\alpha) e^{-i \mu} \cos \mu(\mu \in \mathbb{R},|\mu| \leq$ $\pi / 2,0 \leq \alpha<1), A=1, B=-1$, and $e^{i \theta}=x$ in Theorem 4 , we obtain the result obtained by Ravichandran et al. [7, Theorem 1.2 with $p=1]$.

Theorem 6. If $f(z) \in \Sigma$, then $f(z) \in \sum \mathscr{K}_{\lambda}[b ; A, B]$ if and only if

$$
\begin{array}{r}
z\left[f(z) * \frac{1-3 z+2[(\lambda-1) M+(\lambda+1)] z^{2}}{z(1-z)^{3}}\right] \neq 0 \\
\text { for } z \in \mathbb{U},
\end{array}
$$

where $M=M_{\theta}=\left(e^{-i \theta}+B\right) /(A-B) b, \theta \in[0,2 \pi)$, and also $M=0$.

Proof. Putting

$$
g(z)=\frac{1-[(\lambda-1) M+(\lambda+1)] z}{z(1-z)^{2}},
$$

then

$$
-z g^{\prime}(z)=\frac{1-3 z+2[(\lambda-1) M+(\lambda+1)] z^{2}}{z(1-z)^{3}} .
$$

From (12) and using the identity

$$
\left[-z f^{\prime}(z)\right] * g(z)=f(z) *\left[-z g^{\prime}(z)\right],
$$

we obtain the required result from Theorem 4 .

Remark 7. (i) Putting $\lambda=0$ in Theorem 6, we obtain the result obtained by Bulboacă et al. [5, Theorem 2].

(ii) Putting $\lambda=0, b=1$, and $e^{i \theta}=x$ in Theorem 4, we obtain the result obtained by Ponnusamy [8, Theorem 2.2].

Theorem 8. If $f(z) \in \Sigma$, then $f(z) \in \Sigma \mathcal{S}_{\lambda, v}^{*}[b ; A, B]$ if and only if

$$
\begin{aligned}
& 1+\sum_{k=1}^{\infty} \frac{(-1)^{k}(1-k \lambda) \Gamma(v+1)}{4^{k} \Gamma(k+1) \Gamma(k+v+1)} a_{k} z^{k} \neq 0, \\
& 1+\sum_{k=1}^{\infty}(-1)^{k} \Gamma(v+1) \\
& \quad \cdot\left[\frac{(1-k \lambda)(A-B) b}{4^{k}(A-B) b \Gamma(k+1) \Gamma(k+v+1)}\right. \\
& \left.\quad-\frac{-k(\lambda-1)\left(e^{-i \theta}+B\right)}{4^{k}(A-B) b \Gamma(k+1) \Gamma(k+v+1)}\right] a_{k} z^{k} \neq 0,
\end{aligned}
$$

for all $\theta \in[0,2 \pi)$.

Proof. If $f(z) \in \Sigma$, from Theorem 4, we have $f(z) \in$ $\sum \mathcal{S}_{\lambda, v}^{*}[b ; A, B]$ if and only if

$$
z\left[\left(\mathscr{L}_{v} f\right)(z) * \frac{1-[(\lambda-1) M+(\lambda+1)] z}{z(1-z)^{2}}\right] \neq 0
$$

for $z \in \mathbb{U}$,

where $M=M_{\theta}=\left(e^{-i \theta}+B\right) /(A-B) b, \theta \in[0,2 \pi)$, and also $M=0$. Since

$$
\frac{1-(\lambda+1) z}{z(1-z)^{2}}=\frac{1}{z}+\sum_{k=1}^{\infty}(1-k \lambda) z^{k-1}, \quad z \in \mathbb{U}^{*}
$$

it is easy to show that (28) holds for $M=0$ if and only if (26) holds. Also,

$$
\begin{aligned}
& \frac{1-[(\lambda-1) M+(\lambda+1)] z}{z(1-z)^{2}} \\
& =\frac{1}{z}+\sum_{k=1}^{\infty}[(1-k \lambda)-k(\lambda-1) M] z^{k-1}, \quad z \in \mathbb{U}^{*} ;
\end{aligned}
$$

we may easily check that (28) is equivalent to (27). This completes the proof of Theorem 8 . 
Theorem 9. If $f(z) \in \Sigma$, then $f(z) \in \Sigma \mathscr{K}_{\lambda, v}[b ; A, B]$ if and only if

$$
\begin{aligned}
& 1+\sum_{k=1}^{\infty} \frac{(-1)^{k}(k \lambda-1)(k-1) \Gamma(v+1)}{4^{k} \Gamma(k+1) \Gamma(k+v+1)} a_{k} z^{k} \neq 0, \\
& 1+\sum_{k=1}^{\infty}(-1)^{k}(k-1) \Gamma(v+1) \\
& \quad \cdot\left[\frac{(k \lambda-1)(A-B) b}{4^{k}(A-B) b \Gamma(k+1) \Gamma(k+v+1)}\right. \\
& \left.+\frac{k(\lambda-1)\left(e^{-i \theta}+B\right)}{4^{k}(A-B) b \Gamma(k+1) \Gamma(k+v+1)}\right] a_{k} z^{k} \neq 0,
\end{aligned}
$$

for all $\theta \in[0,2 \pi)$.

Proof. If $f(z) \in \Sigma$, from Theorem 6, we have $f(z) \in$ $\sum \mathscr{K}_{\lambda, v}[b ; A, B]$ if and only if

$$
\begin{aligned}
& z\left[\left(\mathscr{L}_{v} f\right)(z) * \frac{1-3 z+2[(\lambda-1) M+(\lambda+1)] z^{2}}{z(1-z)^{3}}\right] \\
& \quad \neq 0 \text { for } z \in \mathbb{U},
\end{aligned}
$$

where $M=M_{\theta}=\left(e^{-i \theta}+B\right) /(A-B) b, \theta \in[0,2 \pi)$, and also $M=0$. Since

$$
\begin{array}{r}
\frac{1-3 z+2(\lambda+1) z^{2}}{z(1-z)^{3}}=\frac{1}{z}+\sum_{k=1}^{\infty}(k \lambda-1)(k-1) z^{k-1}, \\
z \in \mathbb{U}^{*},
\end{array}
$$

it is easy to show that (33) holds for $M=0$ if and only if (31) holds. Also,

$$
\begin{aligned}
& \frac{1-3 z+2[(\lambda-1) M+(\lambda+1)] z^{2}}{z(1-z)^{3}} \\
& \quad=\frac{1}{z}+\sum_{k=1}^{\infty}(k-1)[k(\lambda-1) M+(k \lambda-1)] z^{k-1} ;
\end{aligned}
$$

for $z \in \mathbb{U}^{*}$, we may easily check that (33) is equivalent to (32). This completes the proof of Theorem 9.

Unless otherwise mentioned, we assume throughout the remainder of this section that $v$ is a real number $(v>-1)$.

Theorem 10. If $f(z) \in \sum$ satisfies inequalities

$$
\begin{aligned}
& \sum_{k=1}^{\infty}|k \lambda-1| \frac{\Gamma(v+1)}{4^{k} \Gamma(k+1) \Gamma(k+v+1)}\left|a_{k}\right|<1, \\
& \sum_{k=1}^{\infty} \frac{[(|1-k \lambda|)(A-B)|b|+k(1-\lambda)(1+|B|)] \Gamma(v+1)}{4^{k} \Gamma(k+1) \Gamma(k+v+1)}\left|a_{k}\right| \\
& \quad<(A-B)|b|,
\end{aligned}
$$

then $f(z) \in \sum \mathcal{S}_{\lambda, v}^{*}[b ; A, B]$.
Proof. We have

$$
\begin{aligned}
\mid & -\sum_{k=1}^{\infty} \frac{(-1)^{k}(k \lambda-1) \Gamma(v+1)}{4^{k} \Gamma(k+1) \Gamma(k+v+1)} a_{k} z^{k} \mid \\
& \geq 1-\left|\sum_{k=1}^{\infty} \frac{(-1)^{k}(k \lambda-1) \Gamma(v+1)}{4^{k} \Gamma(k+1) \Gamma(k+v+1)} a_{k} z^{k}\right| \\
& \geq 1-\sum_{k=1}^{\infty}|k \lambda-1| \frac{\Gamma(v+1)}{4^{k} \Gamma(k+1) \Gamma(k+v+1)}\left|a_{k}\right|\left|z^{k}\right| \\
& \geq 1-\sum_{k=1}^{\infty}|k \lambda-1| \frac{\Gamma(v+1)}{4^{k} \Gamma(k+1) \Gamma(k+v+1)}\left|a_{k}\right| \\
& >0, \quad \text { for } z \in \mathbb{U},
\end{aligned}
$$

which implies inequality (36). Also,

$$
\begin{aligned}
\mid & +\sum_{k=1}^{\infty}(-1)^{k} \Gamma(v+1) \\
& \cdot\left[\frac{(1-k \lambda)(A-B) b}{4^{k}(A-B) b \Gamma(k+1) \Gamma(k+v+1)}\right. \\
& \left.-\frac{k(\lambda-1)\left(e^{-i \theta}+B\right)}{4^{k}(A-B) b \Gamma(k+1) \Gamma(k+v+1)}\right] a_{k} z^{k} \mid \geq 1 \\
& -\mid \sum_{k=1}^{\infty}(-1)^{k} \Gamma(v+1)
\end{aligned}
$$$$
\cdot\left[\frac{(1-k \lambda)(A-B) b}{4^{k}(A-B) b \Gamma(k+1) \Gamma(k+v+1)}\right.
$$$$
\left.-\frac{k(\lambda-1)\left(e^{-i \theta}+B\right)}{4^{k}(A-B) b \Gamma(k+1) \Gamma(k+v+1)}\right] a_{k} z^{k} \mid \geq 1
$$$$
-\sum_{k=1}^{\infty} \Gamma(v+1)
$$

$$
\cdot\left[\frac{|1-k \lambda|(A-B)|b|}{4^{k}(A-B)|b| \Gamma(k+1) \Gamma(k+v+1)}\right.
$$$$
\left.+\frac{k(1-\lambda)(1+|B|)}{4^{k}(A-B)|b| \Gamma(k+1) \Gamma(k+v+1)}\right]\left|a_{k}\right|\left|z^{k}\right|
$$$$
\geq 1-\sum_{k=1}^{\infty} \Gamma(v+1)
$$$$
\cdot\left[\frac{|1-k \lambda|(A-B)|b|}{4^{k}(A-B)|b| \Gamma(k+1) \Gamma(k+v+1)}\right.
$$$$
\left.+\frac{k(1-\lambda)(1+|B|)}{4^{k}(A-B)|b| \Gamma(k+1) \Gamma(k+v+1)}\right]\left|a_{k}\right|>0 \text {, }
$$

for $z \in \mathbb{U}$, 
which implies inequality (37). Thus, the proof of Theorem 10 is completed.

Using similar arguments to those in the proof of Theorem 10, we obtain the following theorem.

Theorem 11. If $f(z) \in \Sigma$ satisfies inequalities

$$
\begin{aligned}
& \sum_{k=1}^{\infty}|k \lambda-1| \frac{(k-1) \Gamma(v+1)}{4^{k} \Gamma(k+1) \Gamma(k+v+1)}\left|a_{k}\right|<1, \\
& \sum_{k=1}^{\infty}(k-1) \Gamma(v+1)\left[\frac{(|k \lambda-1|)(A-B)|b|}{4^{k} \Gamma(k+1) \Gamma(k+v+1)}\right. \\
& \left.\quad+\frac{k(1-\lambda)(1+|B|)}{4^{k} \Gamma(k+1) \Gamma(k+v+1)}\right]\left|a_{k}\right|<(A-B)|b|,
\end{aligned}
$$

then $f(z) \in \sum \mathscr{K}_{\lambda, v}[b ; A, B]$.

Now, using the method due to Ahuja [9], we will prove the containment relations for the subclasses $\sum \mathcal{S}_{\lambda, v}^{*}[b ; A, B]$ and $\sum \mathscr{K}_{\lambda, v}[b ; A, B]$.

Theorem 12. For $v>-1$, we have $\sum \mathcal{S}_{\lambda, v+1}^{*}[b ; A, B] \quad \mathrm{C}$ $\sum \mathcal{S}_{\lambda, v}^{*}[b ; A, B]$.

Proof. Since $f(z) \in \sum \mathcal{S}_{\lambda, v+1}^{*}[b ; A, B]$, we see from Theorem 8 that

$$
\begin{aligned}
& 1+\sum_{k=1}^{\infty} \frac{(-1)^{k}(1-k \lambda) \Gamma(v+2)}{4^{k} \Gamma(k+1) \Gamma(k+v+2)} a_{k} z^{k} \neq 0, \\
& 1+\sum_{k=1}^{\infty}(-1)^{k} \Gamma(v+2) \\
& \quad \cdot\left[\frac{(1-k \lambda)(A-B) b}{4^{k}(A-B) b \Gamma(k+1) \Gamma(k+v+2)}\right. \\
& \left.\quad-\frac{k(\lambda-1)\left(e^{-i \theta}+B\right)}{4^{k}(A-B) b \Gamma(k+1) \Gamma(k+v+2)}\right] a_{k} z^{k} \neq 0 .
\end{aligned}
$$

We can write (41) as

$$
\begin{aligned}
& {\left[1+\sum_{k=1}^{\infty} \frac{v+1}{k+v+1} z^{k}\right] *[1} \\
& \left.+\sum_{k=1}^{\infty} \frac{(-1)^{k}(1-k \lambda) \Gamma(v+1)}{4^{k} \Gamma(k+1) \Gamma(k+v+1)} a_{k} z^{k}\right] \neq 0, \\
& {\left[1+\sum_{k=1}^{\infty} \frac{v+1}{k+v+1} z^{k}\right] *\left[1+\sum_{k=1}^{\infty}(-1)^{k} \Gamma(v+1)\right.} \\
& \cdot\left[\frac{(1-k \lambda)(A-B) b}{4^{k}(A-B) b \Gamma(k+1) \Gamma(k+v+1)}\right. \\
& \left.\left.-\frac{k(\lambda-1)\left(e^{-i \theta}+B\right)}{4^{k}(A-B) b \Gamma(k+1) \Gamma(k+v+1)}\right] a_{k} z^{k}\right] \neq 0,
\end{aligned}
$$

since

$$
\begin{aligned}
& {\left[1+\sum_{k=1}^{\infty} \frac{v+1}{k+v+1} z^{k}\right] *\left[1+\sum_{k=1}^{\infty} \frac{k+v+1}{v+1} z^{k}\right]} \\
& \quad=1+\sum_{k=1}^{\infty} z^{k}
\end{aligned}
$$

By using the property, if $f \neq 0$ and $g * h \neq 0$, then $f *(g * h) \neq$ 0 ; (42) can be written as

$$
\begin{aligned}
& 1+\sum_{k=1}^{\infty} \frac{(-1)^{k}(1-k \lambda) \Gamma(v+1)}{4^{k} \Gamma(k+1) \Gamma(k+v+1)} a_{k} z^{k} \neq 0, \\
& 1+\sum_{k=1}^{\infty}(-1)^{k} \Gamma(v+1) \\
& \quad \cdot\left[\frac{(1-k \lambda)(A-B) b}{4^{k}(A-B) b \Gamma(k+1) \Gamma(k+v+1)}\right. \\
& \left.\quad-\frac{k(\lambda-1)\left(e^{-i \theta}+B\right)}{4^{k}(A-B) b \Gamma(k+1) \Gamma(k+v+1)}\right] a_{k} z^{k} \neq 0,
\end{aligned}
$$

which means that $f(z) \in \sum \mathcal{S}_{\lambda, v}^{*}[b ; A, B]$. This completes the proof of Theorem 12 .

Using the same arguments as in the proof of Theorem 12, we obtain the following theorem.

Theorem 13. For $v>-1$, we have $\sum \mathscr{K}_{\lambda, v+1}[b ; A, B] \subset$ $\sum \mathscr{K}_{\lambda, v}[b ; A, B]$.

\section{Conflict of Interests}

The authors declare that there is no conflict of interests regarding the publication of this paper.

\section{References}

[1] S. S. Miller and P. T. Mocanu, Differential Subordinations: Theory and Applications, vol. 225 of Monographs and Textbooks in Pure and Applied Mathematics, Marcel Dekker, New York, NY, USA, 2000.

[2] G. N. Watson, A Treatise on the Theory of Bessel Functions, Cambridge University Press, Cambridge, UK, 1944.

[3] A. Baricz, Generalized Bessel Functions of the First Kind, vol. 1994 of Lecture Notes in Mathematics, Springer, Berlin, Germany, 2010.

[4] R. Szász and P. A. Kupán, "About the univalence of the Bessel functions," Studia Universitatis Babeş-Bolyai-Series Mathematica, vol. 54, no. 1, pp. 127-132, 2009.

[5] T. Bulboacă, M. K. Aouf, and R. M. El-Ashwah, "Convolution properties for subclasses of meromorphic univalent functions of complex order," Filomat, vol. 26, no. 1, pp. 153-163, 2012.

[6] M. K. Aouf, "Coefficient results for some classes of meromorphic functions," The Journal of Natural Sciences and Mathematics, vol. 27, no. 2, pp. 81-97, 1987. 
[7] V. Ravichandran, S. S. Kumar, and K. G. Subramanian, "Convolution conditions for spirallikeness and convex spirallikenesss of certain p-valent meromorphic functions," Journal of Inequalities in Pure and Applied Mathematics, vol. 5, no. 1, pp. 1-7, 2004.

[8] S. Ponnusamy, "Convolution properties of some classes of meromorphic univalent functions," Proceedings of the Indian Academy of Sciences-Mathematical Sciences, vol. 103, no. 1, pp. 73-89, 1993.

[9] O. P. Ahuja, "Families of analytic functions related to Ruscheweyh derivatives and subordinate to convex functions," Yokohama Mathematical Journal, vol. 41, no. 1, pp. 39-50, 1993. 


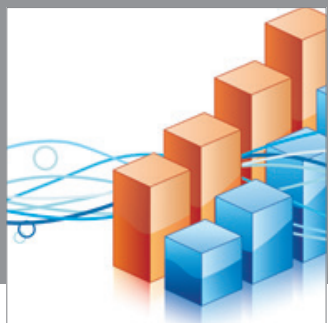

Advances in

Operations Research

mansans

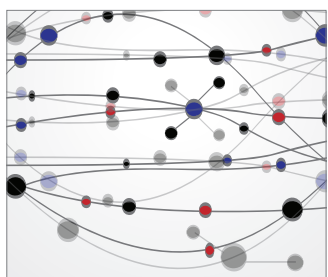

The Scientific World Journal
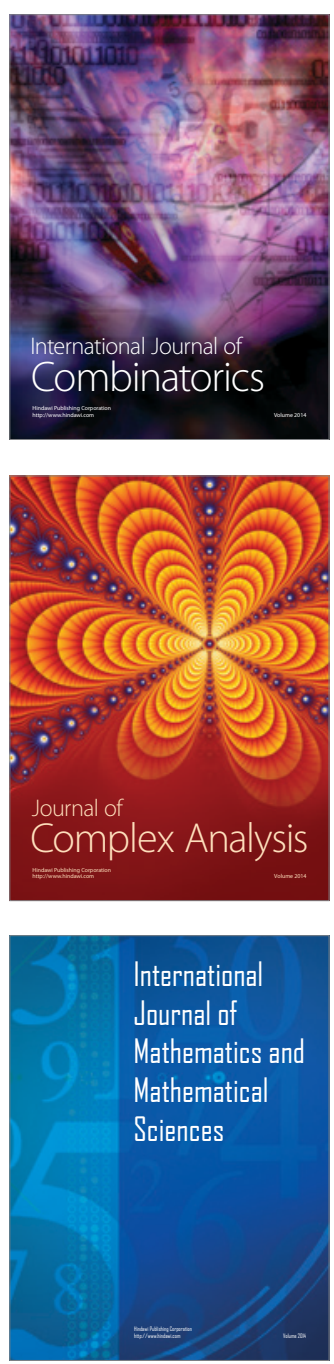
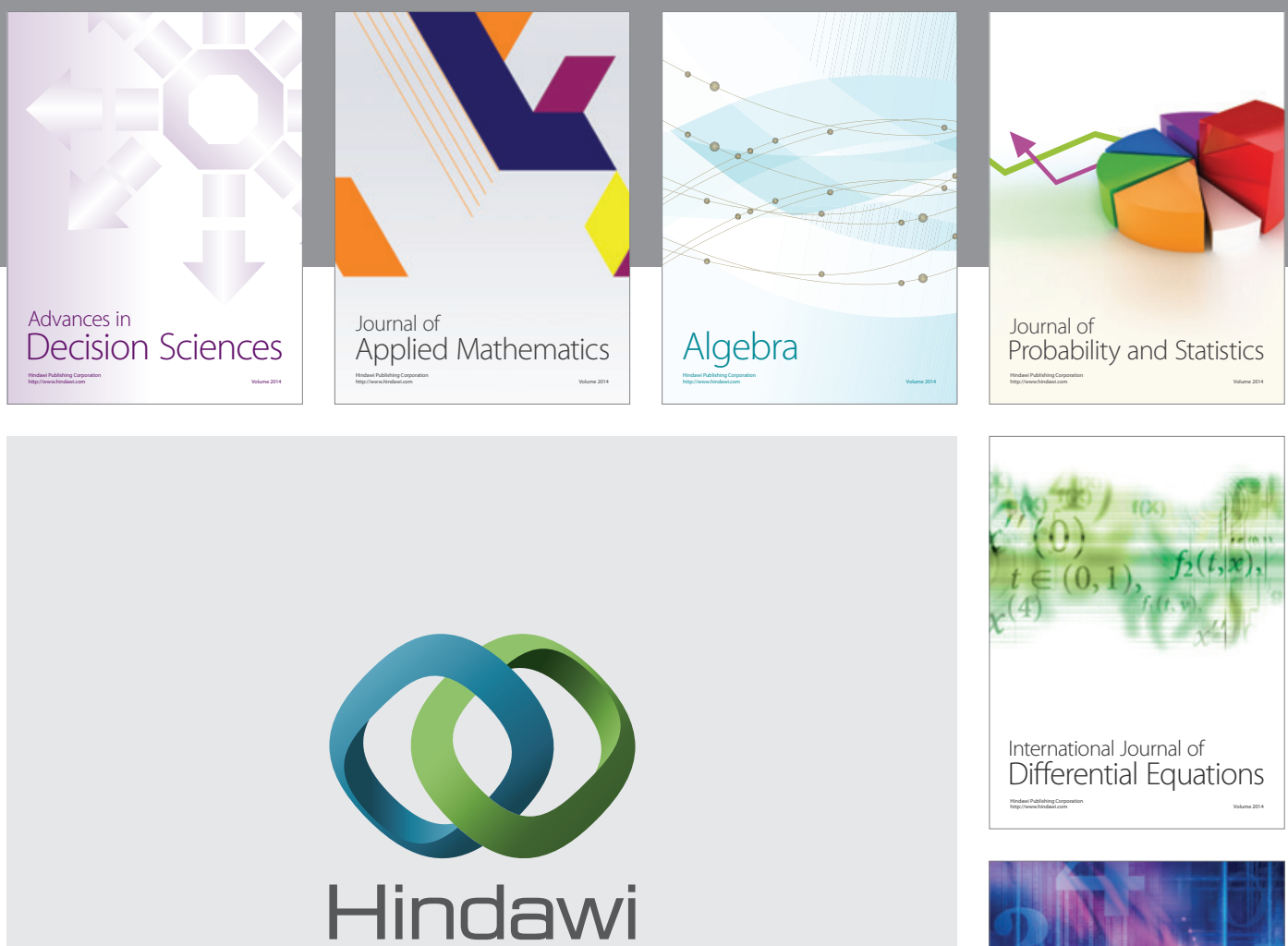

Submit your manuscripts at http://www.hindawi.com
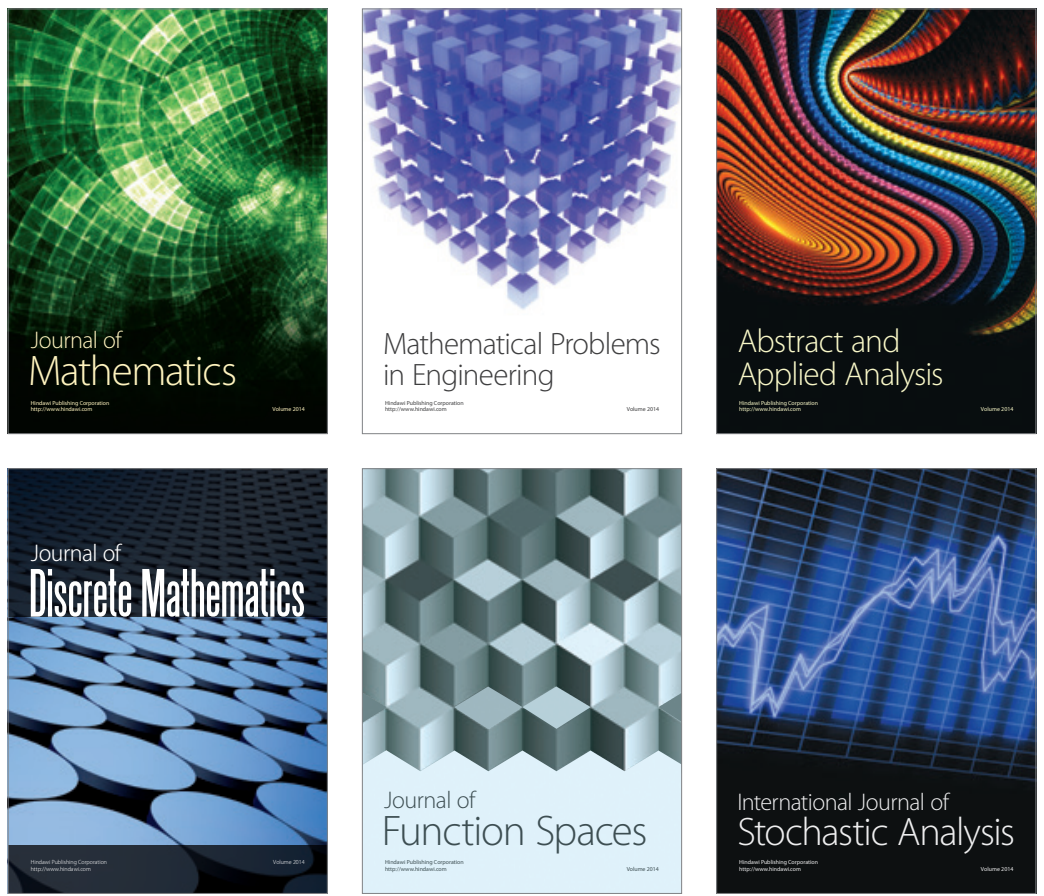

Journal of

Function Spaces

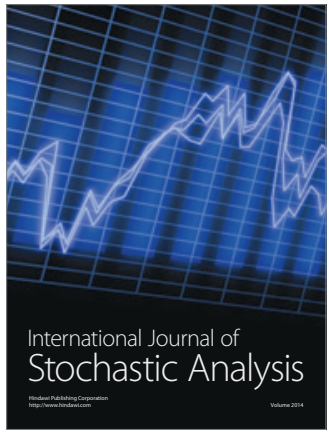

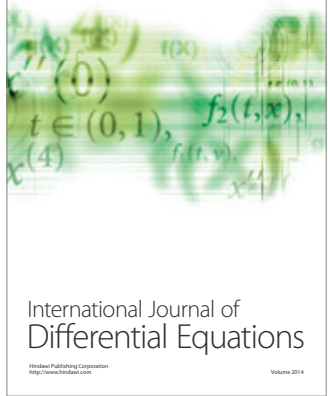
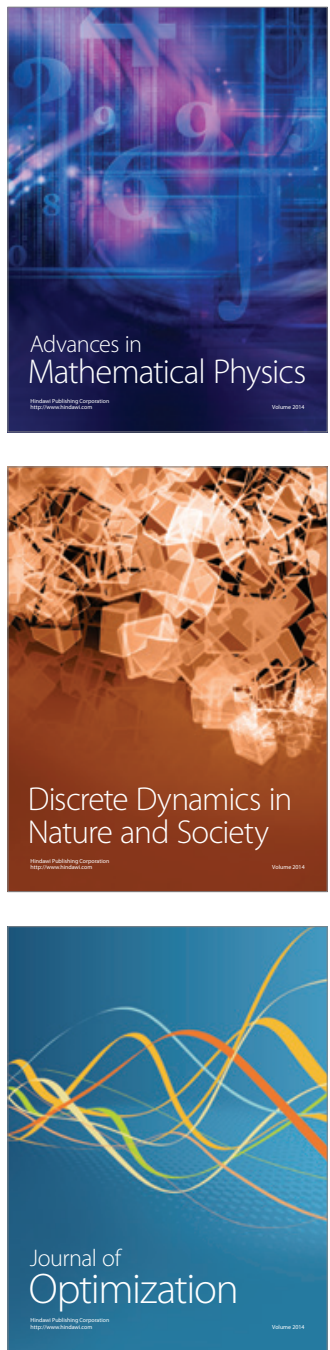